\title{
Mesure des champs de températures et de concentrations des gaz
}

La détermination des températures et des concentrations dans un flux gazeux est essentielle pour le contrôle de processus industriels tels que les combustions, ou dans notre vie courante pour la détection des polluants. Cet article présente une revue critique des méthodes de mesure optiques présentées dans le tableau 1 : méthodes passives utilisant l'émission de rayonnement des gaz, méthodes actives où une excitation extérieure génère sur le volume gazeux un phénomène optique dépendant de la température.

Philippe HERVÉ

Laboratoire LEME

Université Paris Ouest Nanterre la Défense

pherve@u-paris10.fr

> Yannick BAILLY

Institut FEMTO-ST

UMR CNRS 6174

yannick.bailly@univ-fcomte.fr

\section{Comment obtenir}

des mesures absolues locales

de la température

et des concentrations

La température d'un gaz est un phénomène statistique, mais c'est l'aspect macroscopique qui permet de comprendre les propriétés optiques des gaz dues aux transitions entre des niveaux énergétiques, c'est-à-dire la position des raies spectrales. Les chocs entre molécules et l'effet Doppler dû à la température entraînent l'élargissement des raies lorsque la température et la pression augmentent. Ces effets ont freiné l'apparition des méthodes optiques à cause de la difficulté à modéliser ces phénomènes. Dans le domaine industriel, les mesures relatives des températures, autrement dit les repères, ont souvent été considérées comme suffisantes. Des exigences nouvelles sont apparues qui nécessitent de connaître des températures absolues avec précision. Par exemple : les turbines à gaz ont un excellent rendement qui augmente avec la température des gaz. Mais au-delà de $1700^{\circ} \mathrm{C}$, la production de NOx augmente exponentiellement avec la température et il est alors néces- saire de déterminer les profils de température à $10{ }^{\circ} \mathrm{C}$ près.

Les méthodes décrites dans cet article [1] ont déjà été appliquées ou sont en développement préindustriel dans des laboratoires : seront ainsi présentées les méthodes actives les plus utilisées actuellement, puis des méthodes passives et enfin une nouvelle approche conjuguant ces deux types de méthodes.

\section{Méthodes actives}

\section{Diffusion Rayleigh}

Ce type de signal provient de la diffusion de la lumière lors des collisions élastiques entre les phonons et les molécules du gaz.

L'intensité totale diffusée est proportionnelle au nombre de molécules diffusantes. Chaque molécule est caractérisée par une section efficace angulaire de diffusion $\sigma_{R}$ qui dépend des paramètres suivants :

- indice de réfraction $n_{i}$

- longueur d'onde du rayonnement monochromatique incident $\lambda$

- angle $\vartheta$ formé par le vecteur incident $E$ et la direction d'observation de la diffusion Rayleigh.

L'intensité totale de la lumière diffusée est la somme des différentes intensités de la lumière de chaque molécule pour " $m$ " espèces différentes : en fait, on mesure le nombre de particules diffusantes dans un volume donné et par l'équation d'état, si la pression locale est connue, on remonte à la température [2].

La méthode donne de bons résultats, si I'espèce diffusante est unique, sinon dans le cas des réactions de combustion, le problème est indéterminé.

\section{Fluorescence (LIF)}

Elle est actuellement créée par un laser d'où l'appellation LIF (fluorescence induite par laser). Elle est due à la désactivation d'un état excité d'un atome ou d'une molécule par émission lumineuse spontanée à une longueur d'onde supérieure à la longueur d'onde d'excitation (Loi de Stokes) [3]. Le taux de désactivation collisionnelle dépend des températures rotationnelles et vibrationnelles. Si on peut créer une nappe laser dans le volume étudié et l'observer, on obtient alors un champ de températures en deux dimensions.

\section{Diffusion Raman spontanée}

Cette méthode utilise la diffusion inélastique sur les atomes ou sur les molécules. L'onde incidente est diffusée selon deux fréquences $v_{0} \pm v_{\text {Raman }}$. Par exemple pour l'hydrogène, une excitation de la molécule à $532 \mathrm{~nm}$ conduit à l'apparition des raies de Stokes et anti-Stokes qui varient en fonction de la température. La diffusion ayant lieu dans toutes les directions, I'emploi d'une source de forte puissance est nécessaire.

\section{Diffusion Raman cohérente (DRASC)}

C'est la méthode la plus employée en diffusion. On utilise des processus optiques non linéaires à quatre ondes : deux ondes laser $\omega_{1}$ et $\omega_{5}$ sont mélangées dans un milieu possédant un mode de vibration actif en Raman $\omega_{v}$ et deux émissions caractéristiques de la molécule apparaissent (figure 1) : une onde Stokes et une onde anti-Stokes.

L'intensité du signal DRASC est exponentielle en $\chi^{(3)}$ qui dépend de la 
Tableau 1. Panorama des techniques d'analyse des concentrations et/ou des températures de gaz.

\begin{tabular}{|c|c|c|c|c|}
\hline Methode & Principe & Avantages & Inconvénients & Visée \\
\hline Diffusion Rayleigh & Diffusion élastique & Appareillage Simple & $\begin{array}{l}\text { Connaissance préalable } \\
\text { de la composition } \\
\text { du fluide }\end{array}$ & Ponctuelle, 1D, 2D \\
\hline Diffusion Raman & Diffusion inélastique & Appareillage Simple & $\begin{array}{l}\text { Signal faible } \\
\text { Laser haute énergie }\end{array}$ & Ponctuelle, 1D \\
\hline $\begin{array}{l}\text { Fluorescence induite } \\
\text { par laser (LIF) }\end{array}$ & Diffusion inélastique & $\begin{array}{l}\text { Mesure des radicaux } \\
\text { Résolutions spatiale } \\
\text { et temporelle }\end{array}$ & $\begin{array}{l}\text { Laser UV haute énergie } \\
\text { Sensible au quenching } \\
\text { moléculaire }\end{array}$ & Ponctuelle, 1D, 2D, 3D \\
\hline $\begin{array}{l}\text { Diffusion Raman } \\
\text { anti-Stokes cohérente } \\
\text { (DRASC) }\end{array}$ & Diffusion non-linéaire & $\begin{array}{l}\text { Signal intense } \\
\text { Résolution spatiale } \\
\text { Résolution temporelle }\end{array}$ & $\begin{array}{l}\text { Utilisation de plusieurs } \\
\text { sources laser } \\
\text { Phase-matching }\end{array}$ & Ponctuelle \\
\hline $\begin{array}{l}\text { Mélange dégénéré } \\
\text { à quatre ondes (DFWM) }\end{array}$ & Diffusion non-linéaire & $\begin{array}{l}\text { Signal cohérent } \\
\text { Résolution spatiale } \\
\text { et temporelle }\end{array}$ & $\begin{array}{l}\text { Espèces minoritaires } \\
\text { Arrangement optique } \\
\text { Phase-matching }\end{array}$ & Ponctuelle \\
\hline $\begin{array}{l}\text { Spectroscopie } \\
\text { à large bande }\end{array}$ & Emission spontanée & $\begin{array}{l}\text { Mesure passive } \\
\text { Appareillage simple }\end{array}$ & $\begin{array}{l}\text { Emissivité à déterminer } \\
\text { Balayage spatial }\end{array}$ & Intégration sur une ligne \\
\hline $\begin{array}{l}\text { Spectroscopie } \\
\text { d'absorption }\end{array}$ & Transmission & Signal intense & $\begin{array}{l}\text { Transmission perturbée } \\
\text { par le milieu }\end{array}$ & Intégration sur une ligne \\
\hline $\begin{array}{l}\text { Spectroscopie } \\
\text { d'émission }\end{array}$ & Emission spontanée & $\begin{array}{l}\text { Mesure passive } \\
\text { Simplicité appareillage }\end{array}$ & $\begin{array}{l}\text { Inversion } \\
\text { Résolution spatiale }\end{array}$ & Intégration sur une ligne \\
\hline $\begin{array}{l}\text { Spectroscopie } \\
\text { d'émission + LIDAR }\end{array}$ & $\begin{array}{l}\text { Emission spontanée } \\
+ \text { diffusion élastique }\end{array}$ & $\begin{array}{l}\text { Résolution spatiale } \\
\text { et temporelle }\end{array}$ & Energie du laser & $1 \mathrm{D}$ \\
\hline
\end{tabular}

température. Le signal s'exprime sous la forme suivante :

$S=2.2 \cdot 10^{-47} \cdot \omega_{a s}^{4} \mid \chi^{(a)} \cdot\left(-\omega_{a s} \cdot \omega_{1} \omega_{1}-\left.\right|^{2} \cdot P_{1}^{2} P_{2}\right.$ avec $\omega_{\text {as }}$ fréquence de l'onde anti-Stokes et $\chi$ susceptibilité diélectrique du gaz dont le premier terme non linéaire est $\chi^{(3)}$. Les composantes spectrales de la polarisation dépendent des cartographies de température. La figure 2 [4] présente dans le cas d'une flamme hydrogène-air le champ de température obtenu par

Figure 1. Emission d'une molécule active en Raman.
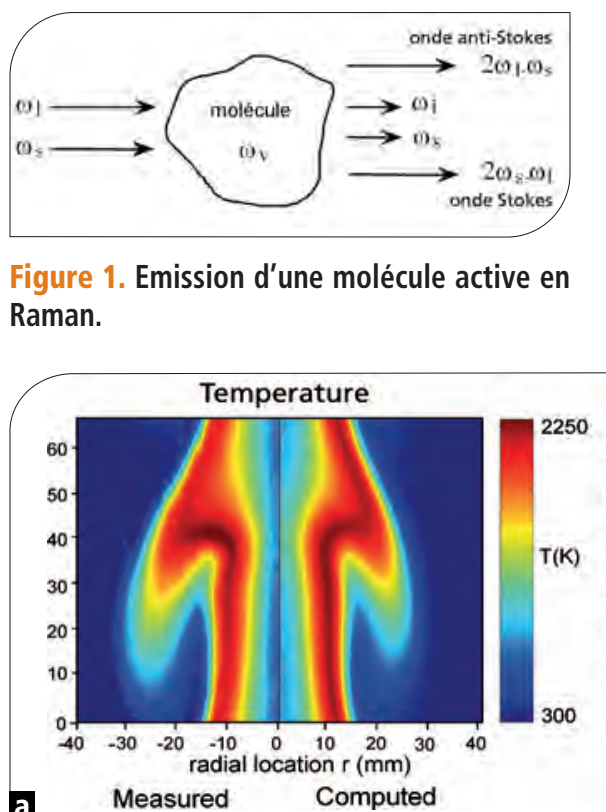

bons résultats sur des concentrations de gaz aux températures atmosphériques, par contre si elles sont utilisées directement sur des gaz chauds le résultat peut être très erroné.

Le principe est voisin de celui des radars, mais avec des longueurs d'onde 10000 fois plus petites en utilisant des sources pulsées telles que des lasers monochromatiques, blancs ou OPO. L'impulsion de la source est diffusée (voir plus haut le paragraphe sur la diffusion Rayleigh) sur la tranche de gaz de profil de concentration $C(x)$ avec des coefficients de rétrodiffusion $\beta$ et d'absorption $K$. De nombreuses variantes ont été utilisées, par exemple le Lidar dit «DIAL » avec une excitation à deux longueurs d'onde, l'une étant sur une raie d'absorption du gaz recherché. Ces méthodes [5] donnent de

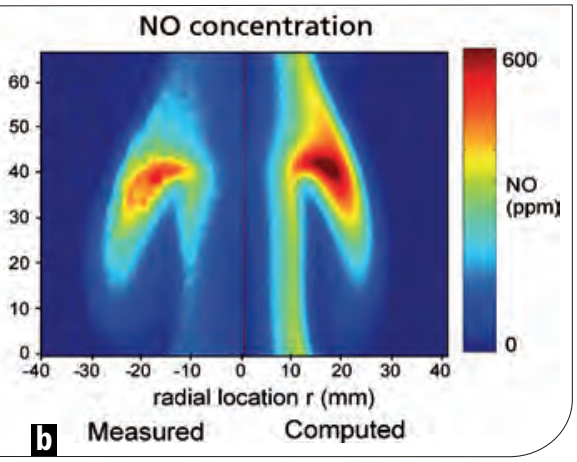

Figure 2. Flamme observée en DRASC (a) et en LIF (b).

\section{Méthodes passives}

Elles sont basées sur l'émission spontanée des gaz.

\section{Méthode de disparition des raies (Kurlbaum, 1906)}

Soit une flamme ensemencée par un traceur tel que le sodium, la flamme étant placée devant un corps noir. Si la température de la flamme est supérieure à celle du corps noir, le doublet du sodium apparaît brillant ; dans le cas contraire, il apparaît noir. L'Onera a développé des appareils basés sur ce principe qui donne une température moyenne de la flamme.

\section{Inversion des spectres d'émission}

Différents auteurs ont utilisé les rapports des intensités des raies d'un gaz pour des mesures de température sur des flammes homogènes. J. Crabol s'est particulièrement intéressé en 1955 à la « tête de bande " du $\mathrm{CO}_{2}$, domaine où le spectre de vibration-rotation présente une sen- 
sibilité à la température et à la pression très prononcée (figure 3).

Depuis 1988, ce travail a été poursuivi au LEME, en coopération avec MBDA Bourges [6] sur la mesure des champs de température dans les statoréacteurs kérosène/air et les super-statoréacteurs hydrogène/air à combustion supersonique.

Le principe des mesures développées au LEME (1988-2010) pour la mesure des profils de température et de concentration est le suivant: le spectromètre placé devant le mélange gazeux mesure la luminance $L$ émise suivant l'axe des $x$ (figure 4).

La luminance perçue par le système de mesure est donnée par l'équation dite de transfert radiatif.

Dans le cas des courtes longueurs d'onde et des milieux chargés en particules, il faut ajouter à l'équation des termes de diffusion. C'est l'inversion de cette équation [7] qui permet d'obtenir simultanément les profils de température et de concentration sur une ligne de mesure.
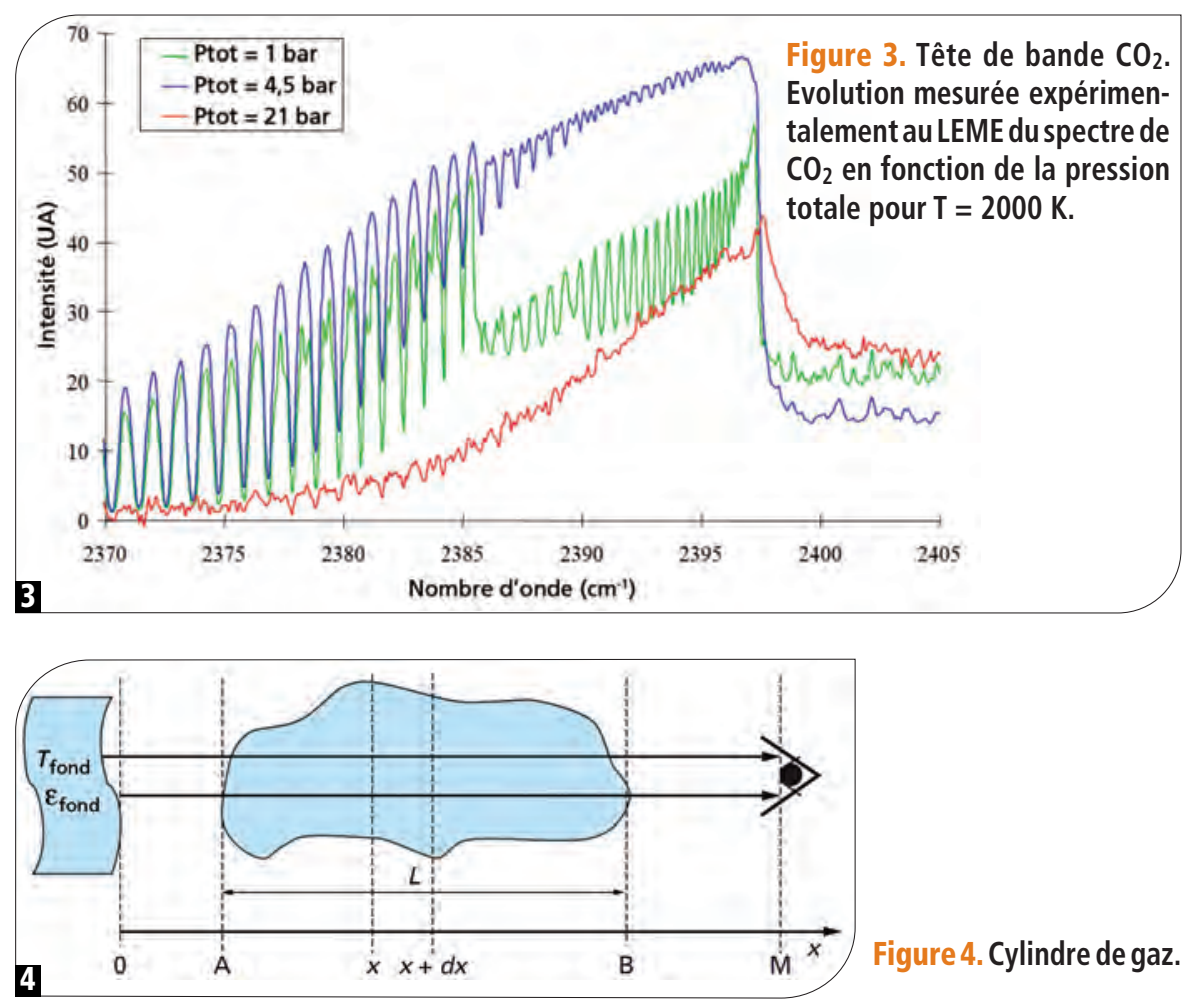

La méthode nécessite la connaissance du facteur d'émission $\varepsilon(\lambda, T, P)$ du gaz

le logiciel FASCODE qui utilise une base de données à pression et température étudié. Les valeurs sont obtenues avec ambiante (HITRAN et HITEMP), et $\gg$

\section{Détecteur thermopile pour l'analyse de gaz} T11262-01

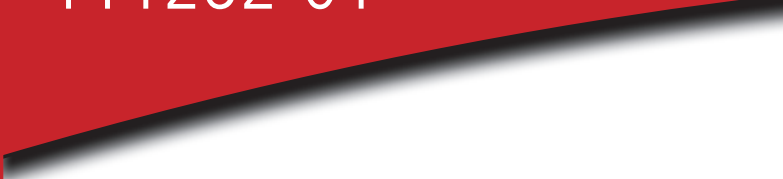

\section{Spécifications :}

- Haute sensibilité sur la bande 3 - $5 \mu \mathrm{m}$

- Boîtier TO-18

- Faible TCR (Temperature Cœfficient of Resistance)

- Très bonne capacité de détection $\left(D^{*}\right)$

\section{Applications:}

- Analyse de plusieurs types de gaz en fonction de votre filtre passe-bande

\section{www.hamamatsu.fr}

infos@hamamatsu.fr - Tél. +33169537100

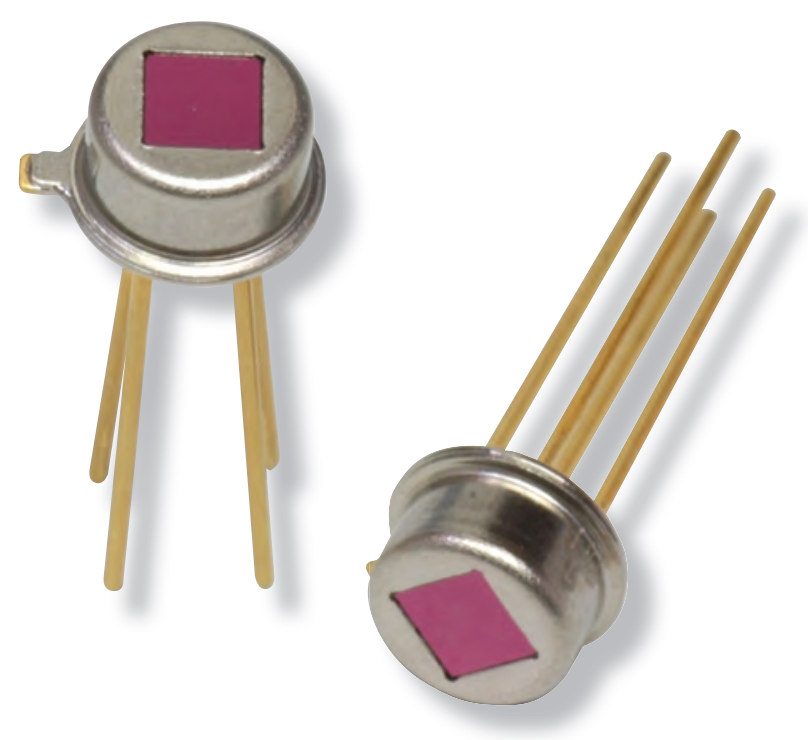


réalise des extrapolations aux hautes températures et pressions. Bien qu'en amélioration régulière, les extrapolations sont parfois erronées et ne prennent pas en compte les transitions moléculaires qui apparaissent à haute température. C'est la raison qui a conduit le LEME et FEMTO-ST à étudier les propriétés spectrales du $\mathrm{CO}_{2}$ et de $\mathrm{H}_{2} \mathrm{O}$ dans les conditions de combustion ou de détonation [8]. La figure 5 montre un exemple typique de résultats obtenus à Farnborough sur l'échappement d'un turboréacteur (Brevet Auxitrol de J. Vally, P. Hervé et al., janvier 1998). La comparaison des mesures optiques effectuées alors en quel ques secondes avec des mesures intrusives effectuées en deux heures par le DSTL anglais a permis de vérifier la méthode ainsi que la précision des résultats obtenus dans des conditions hors laboratoire.

Avec un imageur hyperspectral composé en général d'un spectromètre à transformée de Fourier muni d'une matrice de détecteurs, on obtient en une seule mesure le champ complet de températures et de concentrations

\section{Cas des nuages contenant des particules}

Pour les nuages contenant des particules tels que des aérosols ou des effluents de combustion, la température des particules, souvent voisine de celle des gaz porteurs, peut être mesurée par thermographie bi-spectrale dans l'infrarouge et pour des températures supérieures à $1000{ }^{\circ} \mathrm{C}$ (combustion du charbon pulvérisé, fusées à propergol solide) les mesures seront effectuées de préférence dans I'ultraviolet.

\section{Conjugaison d'une méthode active et d'une méthode passive}

Les méthodes exposées précédemment sont limitées soit à une observation ponctuelle, soit à l'étude de profils simples. Pour la caractérisation de profils complexes, le LEME a conjugué (Brevet Université Paris Ouest de Philippe Hervé, mai 2009) la sensibilité à la température des spectres d'émission et la sensibilité à la concentration et à la localisation
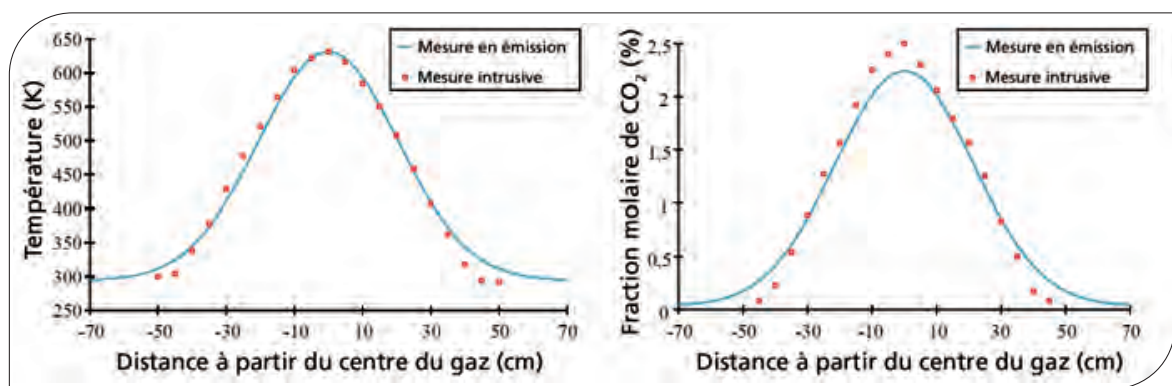

Figure 5. Comparaison des profils de température et de concentration d'un turboréacteur Rolls-Royce obtenus par l'inversion des spectres des têtes de bande du $\mathrm{CO}_{2}$.

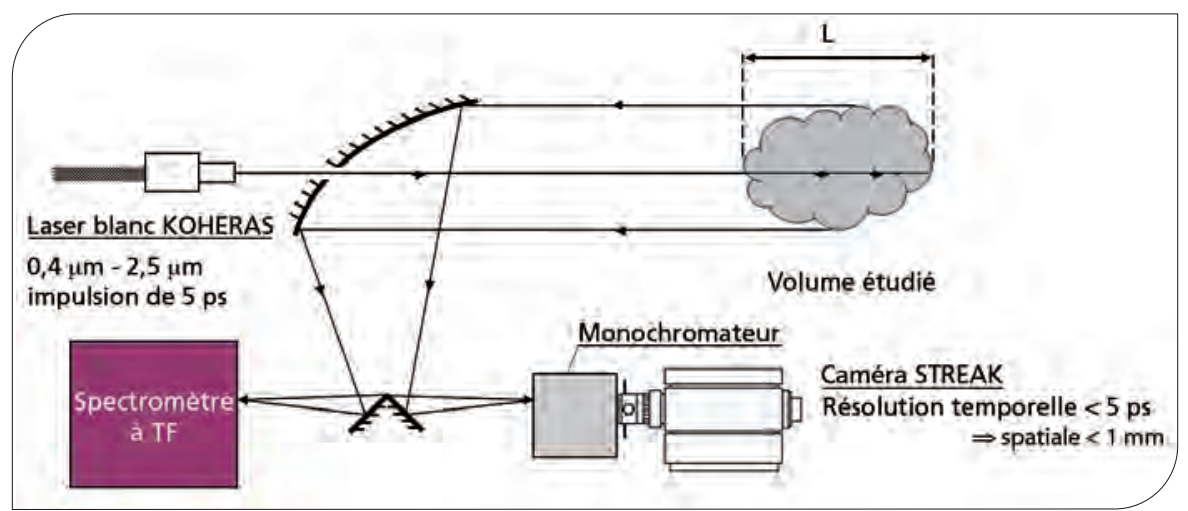

Figure 6. Conjugaison d'un spectre d'émission et du signal d'un Lidar.

spatiale d'un Lidar. Le LEME, FEMTO-ST, I'Onera et MBDA ont obtenu en 2011 le soutien de la DGA (programme REI CELEP) pour développer le système schématisé sur la figure 6 .

Le spectromètre à TF mesure des spectres d'émission. Le laser blanc émet des impulsions de durée 5 picosecondes et la camera STREAK permet de détecter le signal de rétrodiffusion du laser blanc. II faut ensuite inverser le système d'équations en émission et rétrodiffusion pour obtenir les profils de température et de concentrations.

Les premières applications visées sont l'étude des super-statoréacteurs, des turboréacteurs et des moteurs cycliques. Sur un moteur cyclique, on pourra en répétant la mesure avec un spectromètre à TF step-scan obtenir des résolutions de quelques microsecondes, c'est-à-dire de l'ordre du degré de rotation du moteur. En remplaçant le spectromètre à TF par l'imageur développé en cours de développement au LEME/FEMTO-ST ou chez Telops ou Brucker, on obtiendra une vision spatiale des champs de température et de concentration et ceci pour plusieurs gaz simultanément. Les pre- miers résultats sont attendus pour la fin de l'année.

\section{Références}

[1] M. Grisch, P. Gicquel, P. Herve. Mesure des températures dans les gaz. Techniques de l'ingénieur, R2732 (2009)

[2] A. Rousseau, E. Teboul, M.J. van de Sande, J.A.M. van der Mullen. Spatially resolved gas temperature measurements by Rayleigh scattering in a microwave discharge. Plasma Sources Sci. Technol., 11, 47-52 (2002)

[3] W.N. Chen, C.C. Tsao, J.B. Nee. Rayleigh lidar temperature measurements in upper troposphere and lower stratosphere. J. Atmos. Sol.-terr. Phys., 66, 39-49 (2004)

[4] F. Grisch et al. Advanced optical diagnostics applied to dynamic flames and turbulent jets. Aerospace Sci. Technol., 6, 465-479 (2002)

[5] P.H. Flamant. Lidars atmosphériques et météorologiques. Principes fondamentaux. Techniques de l'ingénieur, E4310

[6] J.-P. Minard et al. RAPIERE: An innovative industrial optical measurement system for scramjet flows, in Proc. of the 11th AIAAIAAAF Int. Conf. on Space Planes and Hypersonic Systems and Technologies (2002)

[7] P. Al Khoury, P. Hervé, F. Clement, G. Chavent. Inversion of spectroscopic data of $\mathrm{CO} 2$ radiation, in Proc. of the 4th Int. Conf. on Inverse Problems in Engineering (2002)

[8] V. Bouyer, I. Darbord, P. Hervé, G. Baudin, C. Le Gallic. Shock to detonation of nitrométhane: Time resolved emission spectroscopy measurements. Combust. Flame, 144, 139-150 (2006) 